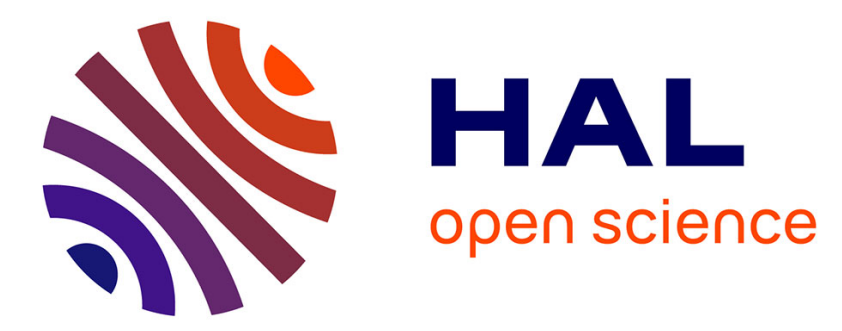

\title{
Design and Kinematic Analysis of a Novel 2-DOF Closed-Loop Mechanism for the Actuation of Machining Robots
}

\author{
Angelica Ginnante, François Leborne, Stéphane Caro, Enrico Simetti, \\ Giuseppe Casalino
}

\section{To cite this version:}

Angelica Ginnante, François Leborne, Stéphane Caro, Enrico Simetti, Giuseppe Casalino. Design and Kinematic Analysis of a Novel 2-DOF Closed-Loop Mechanism for the Actuation of Machining Robots. The ASME 2021 International Design Engineering Technical Conferences \& Computers and Information in Engineering Conference, 45th Mechanisms and Robotics Conference (MR), Aug 2021, Online, United States. hal-03348125

\section{HAL Id: hal-03348125 \\ https://hal.science/hal-03348125}

Submitted on 17 Sep 2021

HAL is a multi-disciplinary open access archive for the deposit and dissemination of scientific research documents, whether they are published or not. The documents may come from teaching and research institutions in France or abroad, or from public or private research centers.
L'archive ouverte pluridisciplinaire HAL, est destinée au dépôt et à la diffusion de documents scientifiques de niveau recherche, publiés ou non, émanant des établissements d'enseignement et de recherche français ou étrangers, des laboratoires publics ou privés. 


\title{
Proceedings of the ASME 2021 International Design Engineering Technical Conferences \& Computers and Information in Engineering Conference IDETC/CIE 2021 \\ August 17-20, 2021, Online, Virtual
}

\section{IDETC2021-70378}

\section{DESIGN AND KINEMATIC ANALYSIS OF A NOVEL 2-DOF CLOSED-LOOP MECHANISM FOR THE ACTUATION OF MACHINING ROBOTS}

\author{
Angelica Ginnante ${ }^{1,2,3}$, François Leborne ${ }^{1}$, Stéphane Caro ${ }^{2}$, Enrico Simetti ${ }^{3}$, Giuseppe Casalino ${ }^{3}$ \\ ${ }^{1}$ Nimbl'Bot, 1 Rue Jean Baptiste Greuze, 33160 Saint-Médard-en-Jalles, France \\ ${ }^{2}$ Laboratoire des Sciences du Numerique de Nantes (LS2N), UMR CNRS 6004, 44300 Nantes, France \\ ${ }^{3}$ University of Genova, DIBRIS, 16145 Genova, Italy \\ Email: aginnante@nimbl-bot.com, fleborne@nimbl-bot.com, \\ stephane.caro@ls2n.fr, Enrico.Simetti@unige.it, pino@dist.unige.it
}

\begin{abstract}
The essential characteristics of machining robots are their stiffness and their accuracy. For machining tasks, serial robots have many advantages such as large workspace to footprint ratio, but they often lack the stiffness required for accurately milling hard materials. One way to increase the stiffness of serial manipulators is to make their joints using closed-loop or parallel mechanisms instead of using classical prismatic and revolute joints. This increases the accuracy of a manipulator without reducing its workspace. This paper introduces an innovative two degrees of freedom closed-loop mechanism and shows how it can be used to build serial robots featuring both high stiffness and large workspace. The design of this mechanism is described through its geometric and kinematic models. Then, the kinematic performance of the mechanism is analyzed, and a serial arrangement of several such mechanisms is proposed to obtain a potential design of a machining robot.
\end{abstract}

\section{INTRODUCTION}

Machining is one of the most critical tasks in the manufacturing industry to transform raw materials into a functional part [1]. Nowadays, mechanical parts are most often machined by Computer Numerical Control (CNC) machines, as such industrial machines feature high stiffness and accuracy. Nevertheless, these machines are generally expensive and do not provide a high versatility [2]. Therefore, Industrial Serial Robots (ISR) started to be investigated for machining tasks, as they can cover larger workspaces, increasing the range of achievable operations [3]. Furthermore, ISRs are also generally cheaper than CNC machines. However, the main drawback of ISRs is their overall lack of stiffness, which leads to an increase in manufacturing errors $[4,5]$. As a matter of fact, classical CNC machines are almost fifty times stiffer than the commercialized ISRs [6].

Despite the overall lack of stiffness of ISRs, some machining tasks were successfully completed by such robots. We can for instance cite polishing [7], grinding [8] and deburring [9]. However, some more challenging tasks, like milling, cause higher efforts on the robot end-effector, leading to machining tool deviation due to the robot compliance [10]. So, the higher the machining forces and moments exerted on the robot end-effector, the larger the point-displacement of the machining tool of an ISR, if the robot compliance is not considered while planning the trajectory and checking the tool motion [3].

In $[11,12]$, the authors listed and analyzed the main sources of errors in robotic machining, which can be caused by the external environment or by the mechanical structure. The latter can be grouped in two main classes: (i) geometrical errors: link lengths, assembling errors, errors in the joint zero values, backlash, (ii) non-geometrical errors: compliance induced errors, measurement errors, environment, control errors, friction. Starting from these sources of errors, two different techniques are described in [11] to reduce the end-effector point-displacement. The first one is offline and based on a predictive compensation of 
machining errors, while the second one is online and uses an external component, named High Dynamic Compensation Mechanism (HDCM), to perform additional positional corrections.

Some other techniques exist to compensate for the manufacturing errors of ISRs. For instance, oversized robots are used to increase the robot stiffness while reducing the efforts on the joints and avoiding singularity regions that affect the positioning accuracy [13]. It should be noted that this approach leads to big and bulky robotic solutions compared to the size of the machining path to follow.

Another way of increasing the stiffness of an ISR is to build it with a series of parallel or closed-loop architectures. These architectures have the advantage of being stiffer than serial ones [14]. Indeed, serial robots usually have a larger workspace to footprint ratio than parallel architectures. On the contrary, parallel robots are stiffer and can hold higher payloads than their serial counterparts. Hybrid manipulators, i.e., serial-parallel robot manipulators, can be an excellent alternative to get both advantages in a single robotic system: good machining accuracy and large workspace to footprint ratio [15].

In [16], the authors assembled several novel two degreesof-freedom mechanisms to build hyper-redundant robots. The investigated concept uses complex and compact designs to optimize the classical joints, namely, revolute and prismatic joints, used in serial and parallel robots. However, the proposed mechanism was optimized for compactness, strength and range of motion, not for stiffness and precision.

The company Nimbl'Bot recently patented a two degreesof-freedom (DoF) mechanism that aims to be a stiff actuator of serial machining robots. The filed patent is [17]. Contrary to the mechanism presented in [16], the mechanism introduced by Nimbl'Bot is optimized for stiffness and positioning accuracy. A first prototype of robot made using the mechanism patented by Nimbl'Bot is being studied, and will be further refered to as "Nimbl'Bot robot". Figure 1 depicts this robot, which turns out to have a large workspace to footprint ratio while remaining stiff and accurate. In fact, it is expected for this robot to have an accuracy of less than $0.1 \mathrm{~mm}$ in a cubic workspace of side $700 \mathrm{~mm}$.

This paper describes and models the patented device [17] and is organized as follows. Section 2 presents a potential use of the mechanism, as well as its innovative design. Section 3 introduces a parameterization of the mechanism, which is used for deriving its geometric model. Section 4 shows how to compute the Jacobian matrix of this mechanism. Section 5 deals with the workspace and kinematic performance analysis of the studied mechanism. Section 6 presents the workspace of a machining robot actuated by ten of the presented mechanisms. Conclusions and future works are presented in Section 7.

\section{OVERVIEW OF THE MACHINING ROBOT AND DE- SCRIPTION OF ITS ACTUATION MECHANISM}

In order to facilitate the understanding of the mechanism under study, this section introduces the potential design of a machining robot actuated by the two-dof mechanism under study. After that, the design of the mechanism itself is presented and explained.

\section{Overview of the Machining Robot}

The robot created by Nimbl'Bot has a particular design and is composed of ten two-DoF mechanisms mounted in series. Figure 1 shows the three regions of the robot, i.e., the shoulder, composed of three actuation mechanisms, the elbow, composed of four actuation mechanisms, and the wrist, composed of three actuation mechanisms.

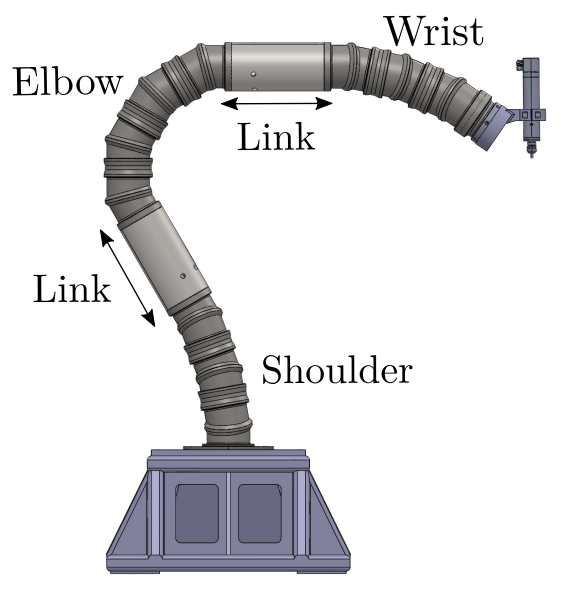

FIGURE 1. POSSIBLE DESIGN FOR A MACHINING ROBOT ACTUATED BY TEN TWO-DOF MECHANISMS MOUNTED IN SERIES. THE SHOULDER AND THE WRIST ARE MADE OF THREE MECHANISMS EACH (ACCOUNTING FOR A TOTAL COVERED SOLID ANGLE OF $\pm \pi / 2$ RADIANS EACH) AND THE ELBOW IS MADE OF FOUR MECHANISMS (SOLID ANGLE OF $\pm 2 \pi / 3$ RADIANS).

Each actuator provides two degrees of freedom. As a result, the robot in Fig. 1 has twenty-DoF. A robot is defined as redundant when it has more DoF than the required number of DoF to perform the desired task [18]. Namely, when the dimension of its actuation vector $\mathbf{q}$ is greater than the dimension of the vector $\mathbf{X}$ that describes the task [19]. Then, a manipulator is said to be hyper-redundant when the size of $\mathbf{q}$ is much greater than $\mathbf{X}$. Consequently, the end-effector can reach the desired pose (position and orientation) with an infinite number of robot postures. Here, 
the Nimbl'Bot robot is hyper-redundant since its twenty-DoF are much more than enough to achieve a task requiring six-DoF, e.g., the placement of the machining tool with the proper position and orientation.

The main goal of a such robot is to ensure high precision during machining tasks, avoiding the use of an oversized design. Due to the stiffness of the mechanism, the robot can follow the desired path with sufficient accuracy. Furthermore, thanks to its high kinematic redundancy degree, the robot is suitable for working in cluttered environments. However, solving the inverse kinematic model of a hyper-redundant robot is challenging [20].

\section{Description of the Actuation Mechanism}

The machining robot described in the previous section is actuated through the novel two-DoF mechanism, which is the main focus of this paper. This actuation system is composed of two linked kinematic chains, making it behave like a parallel mechanism. It provides two coupled rotational motions that can be decoupled by proper control of the two embedded motors. In the rest of this paper, the two-DoF mechanism studied will be referred to as module.

Description of the External Kinematic Chain. The external kinematic chain has four different components, as shown in Fig. 2.

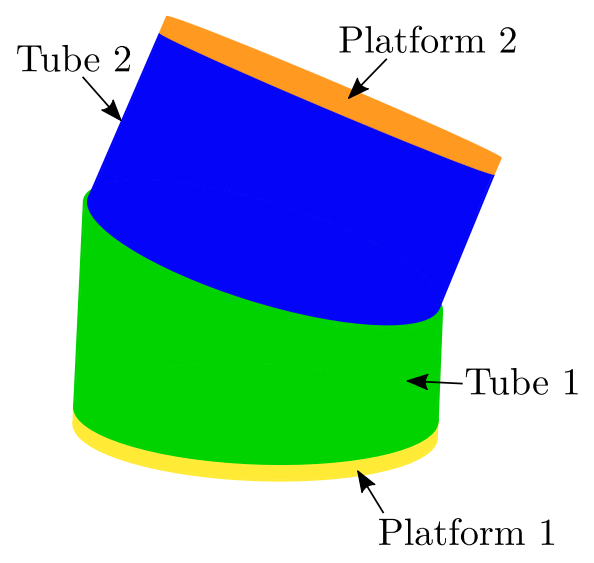

FIGURE 2. EXTERNAL VIEW OF THE MODULE

The fixed base, in yellow, is named Platform 1 and it is considered centered on the origin. Above Platform 1 is a rotating cylinder, in green, named Tube 1 . Tube 1 is a hollow cylinder cut by an oblique plane with a slope $\alpha$. The first motor actuates the component Tube 1. The motor is attached directly to the inner side of Tube 1 . In this way, Tube 1 can rotate around the vertical axis that passes through the center of Platform 1. Tube 2 is placed over Tube 1 and they have the same shape. The second motor actuates Tube 2 and is attached to the inner side of Tube 2 . Tube 2 can rotate around the axis perpendicular to and centered in Platform 2. The external kinematic chain is closed by Platform 2, the moving platform, which is the end-effector of the module.

Tube 1 and Tube 2 are independently actuated. The external kinematic chain is the actuated part of the module.

Description of the Internal Kinematic Chain. The internal kinematic chain has seven components, as shown in Fig. 3. Two of those components also belong to the external kinematic chain, i.e., Platform 1 and Platform 2. Thus, the external and internal kinematic chains make a closed-loop chain.

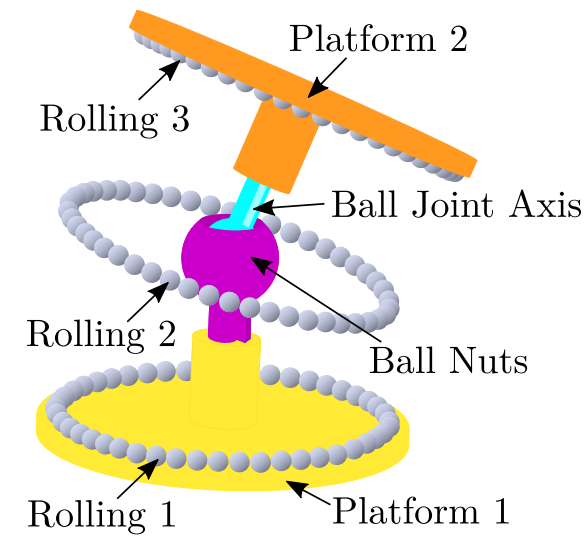

FIGURE 3. INTERNAL VIEW OF THE MODULE

Platform 1 is linked to the component Ball Nuts in purple through a prismatic joint, which prevents internal breaks while the module is actuated. These could occur due to dimensional inaccuracies in the mechanical parts. Following that, there is the Ball Joint Axis in cyan, which forms a constant velocity joint with Ball Nuts. In the end, Ball Joint Axis is linked to Platform 2 through another prismatic joint, again to avoid internal breaks.

One of the most interesting features regarding this innovative module is the constant velocity joint [21], which works like a universal joint, but allows its two ends to rotate at the same velocity. Therefore, fixing Platform 1, especially without applying torsion on it, means forcing no torsion for the whole internal kinematic chain. In this way, the module amounts to a zero-torsion mechanism.

The last three components in the internal kinematic chain are the three rolling lines, Rolling 1, Rolling 2 and Rolling 3. They are composed of a series of balls and are necessary to decouple the rotations of Tube 1, Tube 2 and the internal kinematic chain. 
Consequently, Tube 1 and Tube 2 can independently rotate, leading to an inclination of Platform 2 without a rotation of the latter about its normal axis.

\section{PARAMETERIZATION AND GEOMETRIC MODEL- ING OF THE MODULE}

In [22], two different models were used to describe the module. One model, called Q-model, represents the external kinematic chain of the module. The other one, called T\&A(Tilt\&Azimuth)model, represents the internal kinematic chain. In this section, it is chosen to present a different parameterization that simplifies the computation of the geometric model. The parameters describing the module are two angles, namely the tilt and azimuth angles, that represent the relative orientation of the two platforms of the module. This parameterization and the fact that the mechanism does not introduce torsion between the two platforms motivated the use of the Bonev rotation matrix [23] to describe the relative orientation of the two platforms. This results in the necessity to compute only one matrix for expressing the relative orientation instead of three matrices required by the models introduced in [22]. To complete the geometric modeling of the module, the tilt and azimuth angles are also expressed as functions of the motors coordinates.

\section{Parameterization of a module}

Figure 4 shows the frames attached to each moving part and the relations between each of them. Frames $\langle 0\rangle$ and $\langle 1\rangle$ share the common axis $\vec{z}\left(\overrightarrow{z_{0}} \| \overrightarrow{z_{1}}\right)$, and the rotation angle of $<1>$ around $\overrightarrow{z_{1}}$ is noted $q_{1}\left(\left(\overrightarrow{x_{0}}, \overrightarrow{x_{1}}\right)\right.$ angle $) . q_{1}$ is the first motor coordinate. Then, the rotation angle of $\left\langle 2>\right.$ around $\overrightarrow{z_{2}}$ is noted as $q_{2}$ $\left(\left(\overrightarrow{x_{3}}, \overrightarrow{x_{2}}\right)\right.$ angle $)$. Therefore, $q_{2}$ is the second motor coordinate.

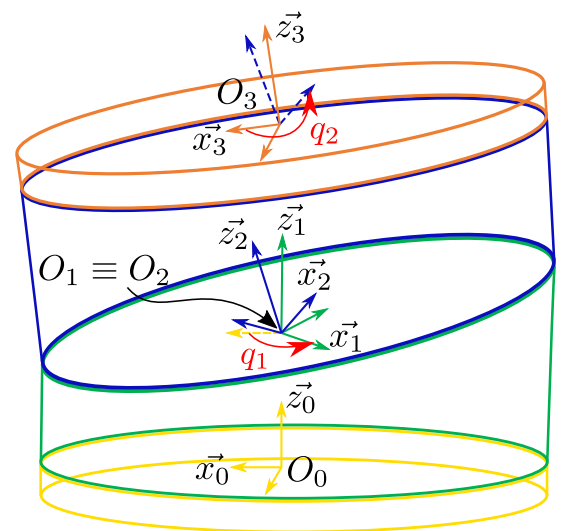

FIGURE 4. EXTERNAL KINEMATIC CHAIN: FRAMES AND ACTUATION ANGLES
Figure 5 shows the zero position of the module. In this position, both $q_{1}$ and $q_{2}$ are equal to 0 . The shortest side of Tube 1 is along axis $\overrightarrow{x_{0}}\left(\overrightarrow{y_{0}}=0\right)$ and the shortest side of Tube 2 is along axis $-\overrightarrow{x_{0}}$ $\left(\overrightarrow{y_{0}}=0\right)$.

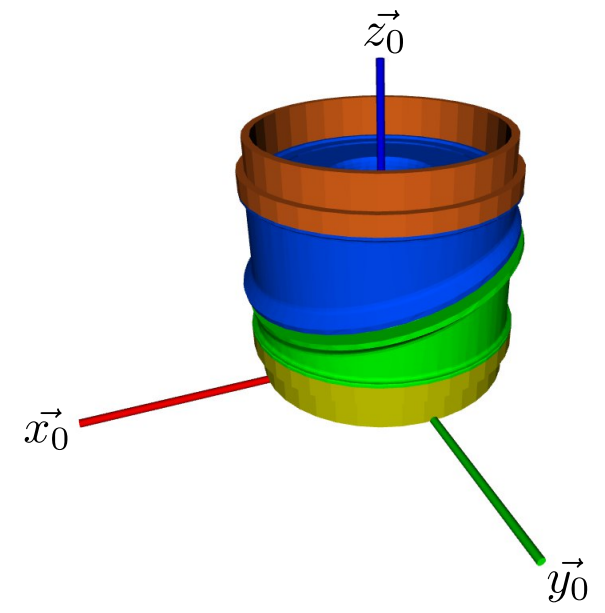

FIGURE 5. HOME POSE OF THE MODULE

Figures 6 and 7 depict the actuated angles $q_{1}$ and $q_{2}$. The variable $q_{1}$ is the rotation angle between Platform Plane $1\left(O_{0}, \overrightarrow{x_{0}}, \overrightarrow{z_{0}}\right)$, in yellow, and Tube Plane $1\left(O_{1}, \overrightarrow{x_{1}}, \overrightarrow{z_{1}}\right)$, in green. Similarly, the variable $q_{2}$ is the rotation angle between Platform Plane 2 $\left(\mathrm{O}_{3}, \overrightarrow{x_{3}} \overrightarrow{z_{3}}\right)$, in orange, and Tube Plane $2\left(\mathrm{O}_{2}, \overrightarrow{x_{2}}, \overrightarrow{z_{2}}\right)$, in blue.

A peculiarity of the module is that both motors can rotate indefinitely without hitting any mechanical stop.

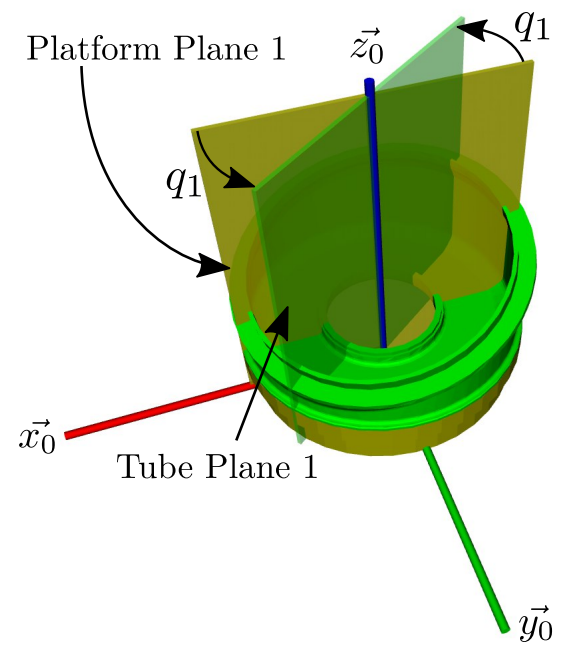

FIGURE 6. ACTUATION VARIABLE $q_{1}$ 


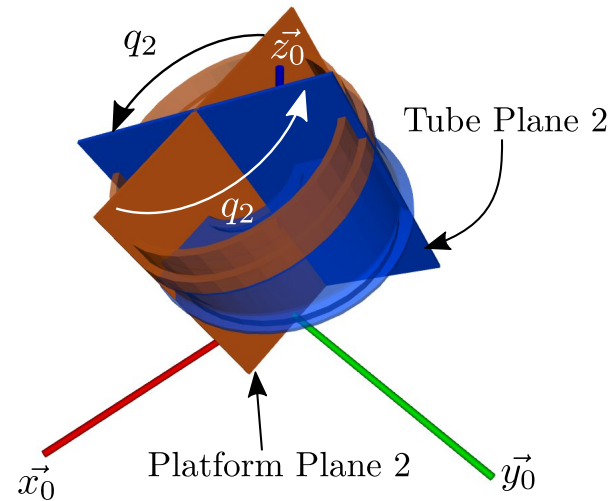

FIGURE 7. ACTUATION VARIABLE $q_{2}$

\section{Zero-Torsion Mechanism}

A zero-torsion mechanism is a mechanism where the ending platform never rotates about its normal axis [24]. To express the zero-torsion rotation matrix, we can start from the Tilt\&Torsion angles notation, defined in [23], and set the torsion to zero. The azimuth, tilt and torsion angles are shown in Fig. 8.

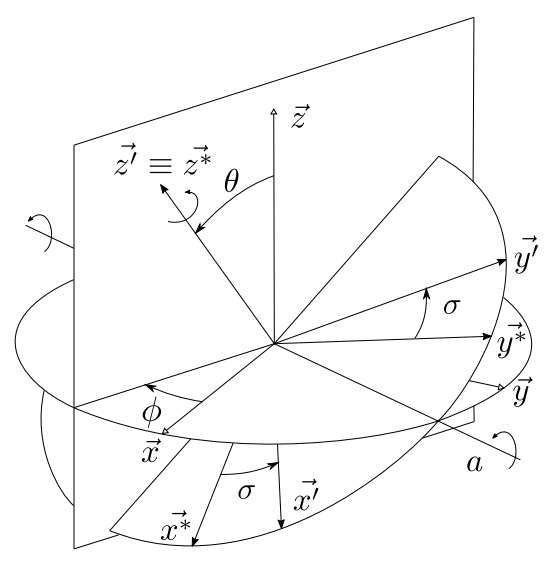

FIGURE 8. TILT AND TORSION ANGLE NOTATION [23]

The Tilt\&Torsion convention involves only two rotation angles, the tilt angle $\theta$ around axis $a$ and the torsion angle $\sigma$ around axis $\overrightarrow{z^{*}}$. Then, the azimuth angle $\phi$ is not a rotation angle anymore. It becomes the angle that defines the orientation of the vertical plane, which is perpendicular to axis $a$. The angles take values as follows: $\theta \in[0, \pi), \sigma \in(-\pi, \pi]$ and $\phi \in(-\pi, \pi]$.

The value of $\sigma$ is set to zero and the rotation matrix of a zerotorsion mechanism is Eq. (1).

$$
\mathbf{R}(\phi, \theta)=\left[\begin{array}{ccc}
\cos ^{2} \phi \cos \theta+\sin ^{2} \phi & \cos \phi \sin \phi(\cos \theta-1) & \cos \phi \sin \theta \\
\sin \phi \cos \phi(\cos \theta-1) & \sin ^{2} \phi \cos \theta+\cos ^{2} \phi & \sin \phi \sin \theta \\
-\sin \theta \cos \phi & -\sin \theta \sin \phi & \cos \theta
\end{array}\right]
$$

\section{Geometric Model of the Module}

Figure 9 shows the Tilt\&Azimuth (T\&A) based geometric model of the module. A series of three revolute joints form the model. The first revolute joint represents the azimuth angle $\phi$ of the module. The second revolute joint is the tilt angle $\theta$. The third revolute joint has the negative value of the azimuth angle $-\phi$ to respect the zero-torsion characteristic of the module. The variable $r$ represents the distance between Platform 1 and the constant velocity joint and between the constant velocity joint and Platform 2.

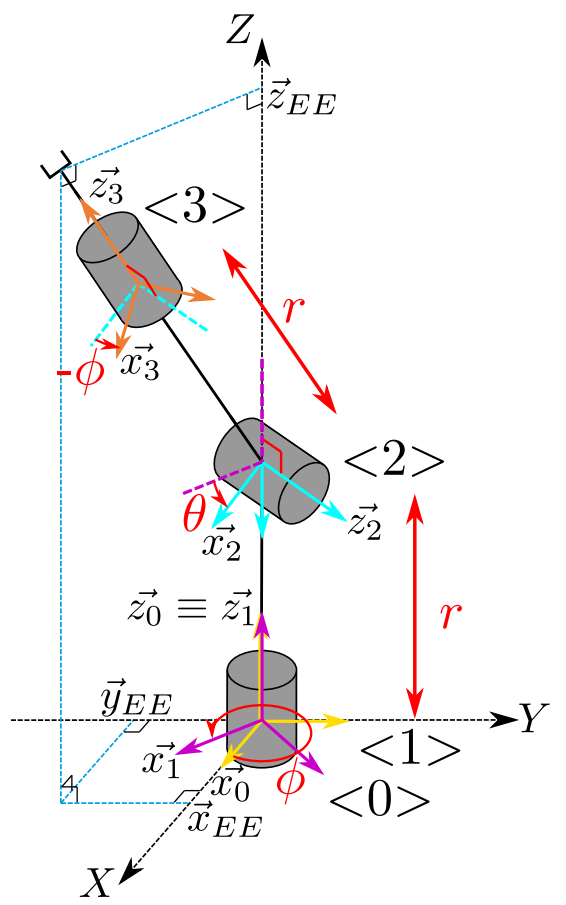

FIGURE 9. TILT AND AZIMUTH MODEL OF THE MODULE

The rotation matrix of the module ${ }^{0} \mathbf{R}_{3}(\phi, \theta)$ is the one expressed in Eq. (1). The multiplication of three rotation matrices generates ${ }^{0} \mathbf{R}_{3}(\phi, \theta)$ : around $\vec{z}_{1}$ of $\phi$ with respect to frame $<0>$, around $\overrightarrow{z_{2}}$ of $\theta$ with respect to frame $<1>$ and around $\overrightarrow{z_{3}}$ of $-\phi$ with respect to frame $<2>$.

The translation vector of the module ${ }^{0} \mathbf{p}_{3}(\phi, \theta, r)$ is the vector 
pointing from point $O_{0}$ to point $O_{3}$ :

$$
{ }^{0} \mathbf{p}_{3}(\phi, \theta, r)=\left[\begin{array}{c}
r \sin \theta \cos \phi \\
r \sin \theta \sin \phi \\
r+r \cos \theta
\end{array}\right]
$$

As a result, the complete homogeneous transformation matrix of the module from frame $<0>$ to frame $<3>$ is expressed as:

$$
{ }^{0} \mathbf{T}_{3}(\phi, \theta, r)=\left[\begin{array}{cc|c|c}
{ }^{0} \mathbf{R}_{3}(\phi, \theta) & { }^{0} \mathbf{p}_{3}(\phi, \theta, r) \\
\hline 0 & 0 & 0 & 1
\end{array}\right]
$$

The azimuth $\phi$ and tilt $\theta$ rotation angles are expressed as functions of the actuation variables $q_{1}$ and $q_{2}$, namely,

$$
\left\{\begin{array}{l}
\phi=\frac{q_{1}+q_{2}-\pi}{2} \\
\theta=\arctan \left(-\frac{2 \tan \alpha \sin \left(\frac{q_{1}-q_{2}}{2}\right)}{1-\tan ^{2} \alpha \sin ^{2}\left(\frac{q_{1}-q_{2}}{2}\right)}\right)
\end{array}\right.
$$

where $\alpha$ is the slope of the oblique planes in Tube 1 and Tube 2 . Similarly, the actuation variables $q_{1}$ and $q_{2}$ are expressed as functions of the azimuth angle $\phi$ and tilt angle $\theta$ as follows:

$$
\left\{\begin{array}{l}
q_{1}=\phi+\arccos \left(-\frac{\cos \alpha(\cos \theta-1)}{\sin \alpha \sin \theta}\right) \\
q_{2}=\phi-\arccos \left(-\frac{\cos \alpha(\cos \theta-1)}{\sin \alpha \sin \theta}\right)+\pi
\end{array}\right.
$$

When the tilt $\theta$ is equal to 0 , the value of the actuation variables is $q_{1}=q_{2}=\phi+\pi / 2$. The azimuth angle $\phi$ gives the orientation along which Platform 2 is tilted. Figure 10 shows the Azimuth Plane.

Figure 11 shows the Tilt Plane, which is parallel to the top of the orange platform and oriented along with the Azimuth Planes. The angle between the $\left(O, \overrightarrow{x_{0}}, \overrightarrow{y_{0}}\right)$ plane and the Tilt Plane is the tilt angle $\theta$.

\section{KINEMATIC MODELING OF THE MODULE}

This section presents the kinematic model of the module based on the parameterization defined in Fig. 9.

The kinematic Jacobian matrix of the module is computed in two steps. First of all, the Jacobian matrix ${ }^{0} \mathbf{J}_{3 \phi \theta}(\phi, \theta, r)$ is calculated. This matrix maps the tilt and azimuth angles time derivatives $[\dot{\phi}, \dot{\theta}]^{\top}$ to the module tip twist $\mathbf{t}=\left[\dot{\mathbf{p}}^{\top}, \boldsymbol{\omega}^{\top}\right]^{\top}$, with $\dot{\mathbf{p}}$ and $\boldsymbol{\omega}$ the linear and angular velocity vectors of the end-effector, respectively.

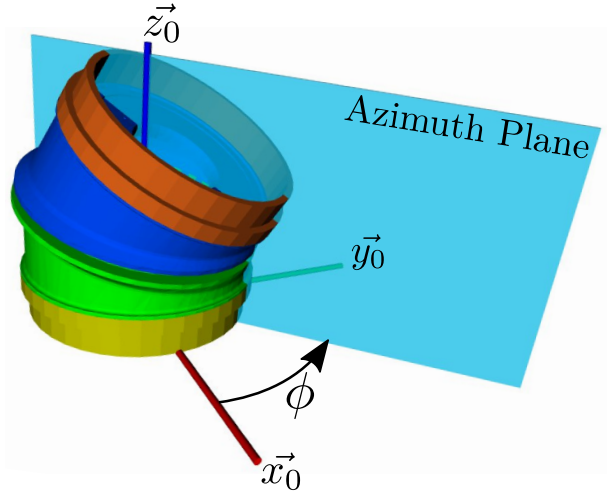

FIGURE 10. AZIMUTH PLANE

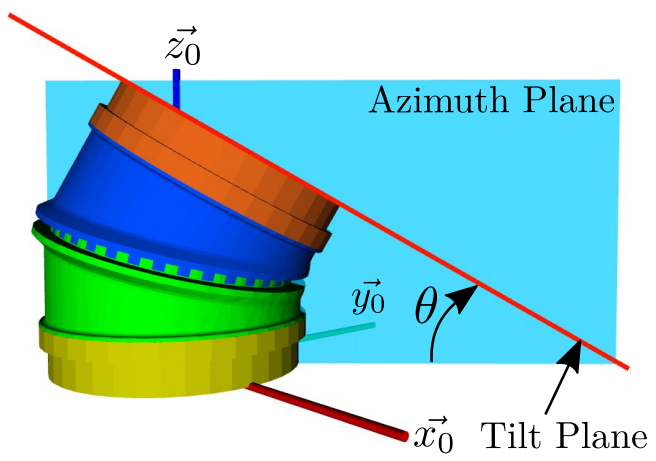

FIGURE 11. TILT AND AZIMUTH PLANES

$$
\mathbf{t}={ }^{0} \mathbf{J}_{3 \phi \theta}(\phi, \theta, r)[\dot{\phi} \quad \dot{\theta}]^{\top}
$$

${ }^{0} \mathbf{J}_{3 \phi \theta}(\phi, \theta, r)$ is expressed as a function of the angle $[\phi, \theta]^{\top}$ as follow:

$$
{ }^{0} \mathbf{J}_{3 \phi \theta}(\phi, \theta, r)=\left[\begin{array}{cc}
-r \sin \phi \sin \theta & r \cos \phi \cos \theta \\
r \cos \phi \sin \theta & r \sin \phi \cos \theta \\
0 & -r \sin \theta \\
-\cos \phi \sin \theta & -\sin \phi \\
-\sin \phi \sin \theta & \cos \phi \\
1-\cos \theta & 0
\end{array}\right]
$$

Then, the Jacobian matrix $\mathbf{J}_{q_{1} q_{2}}$, which maps the motors velocities $\dot{\mathbf{q}}=\left[\dot{q}_{1}, \dot{q}_{2}\right]^{\top}$ to the angles velocities $[\dot{\boldsymbol{\phi}}, \dot{\theta}]^{\top}$, is obtained upon time differentiation of Eq. (4):

$$
\mathbf{J}_{q_{1} q_{2}}\left(q_{1}, q_{2}\right)=\frac{1}{2}\left[\begin{array}{cc}
1 & 1 \\
-c & c
\end{array}\right]
$$


where:

$$
c=\frac{2 \tan \alpha \cos \left(\frac{q_{1}-q_{2}}{2}\right)}{1+\tan ^{2} \alpha \sin ^{2}\left(\frac{q_{1}-q_{2}}{2}\right)}
$$

The complete kinematic Jacobian matrix of the module as a function of the motors coordinates $q_{1}$ and $q_{2}$ is obtained as follows:

$$
\mathbf{t}={ }^{0} \mathbf{J}_{3 \phi \theta} \mathbf{J}_{q_{1} q_{2}} \dot{\mathbf{q}}
$$

\section{PERFORMANCE ANALYSIS OF THE MODULE}

This section presents an analysis of the geometric and kinematic performance of the module. First, the workspace of the module is displayed. Then, the condition number of the Jacobian matrix, expressed in Eq. (10), is computed to assess the dexterity of the mechanism throughout its workspace.

In this section, the geometric parameters $r$ and $\alpha$ are set to $r=$ $0.32 \mathrm{~m}$ and $\alpha=15^{\circ}$, which are the values used for the first module prototype.

\section{Analysis of the Workspace of a Module}

The workspace of the module is a portion of a sphere, Fig. 12. It corresponds to all the positions reached by the center of the upper platform for all the possible values of $q_{1}$ and $q_{2}$ in $[0 ; 2 \pi]$. On this portion of a sphere, each point is reachable by only one orientation for the moving-platform, but in two possible aspects.

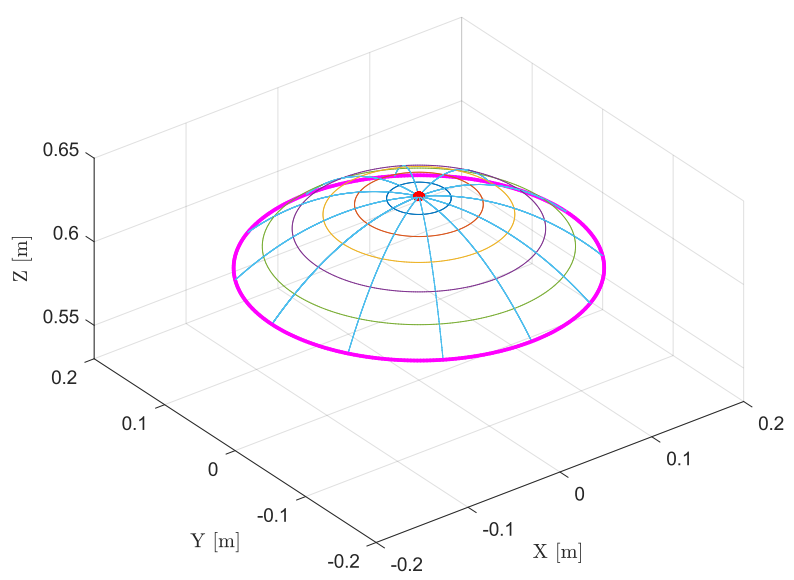

FIGURE 12. 3D VIEW OF THE WORKSPACE OF ONE MODULE
Figure 13 depicts the tilt $\theta$ and azimuth $\phi$ angles as a function of the $X$ and $Y$ Cartesian coordinates of the end-effector. The value of $\phi$ is in $[-\pi, \pi)$ and $\theta$ is in $[-\pi / 6, \pi / 6]$. In fact, the maximum possible value of $|\theta|$ is twice the slope of each Tube $(2 \alpha=\pi / 6)$. The workspace of the module is symmetric around the $\mathrm{Z}$ axis.

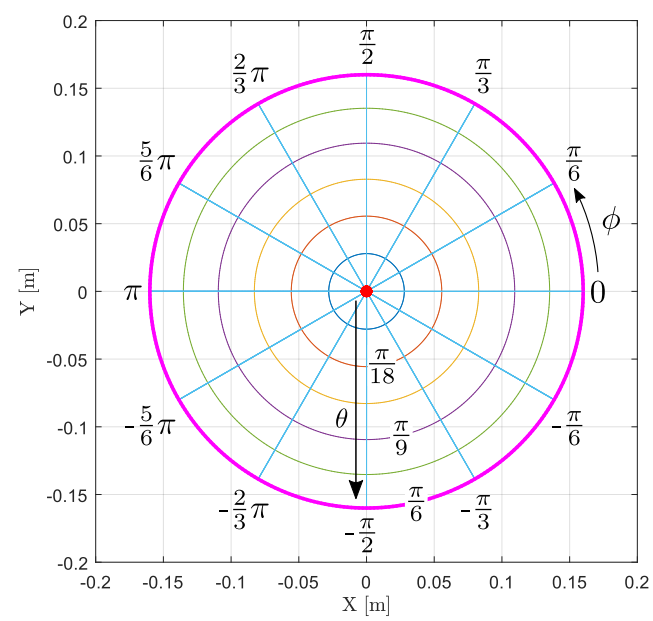

FIGURE 13. 2D VIEW OF THE WORKSPACE OF ONE MODULE

Figure 14 depicts the two areas covered by each aspect of the module, represented in its joint space. The joint space can be divided in two aspects because the inverse kinematics of the module has up to two solutions. In fact, switching the values of $q_{1}$ and $q_{2}$ lead to the same tilt and azimuth angles, which is represented by Fig. 14. This property can easily be verified using Eq. (4).

There are two limit cases where the module does not have strictly two possible aspects leading to a single position of its tip. When the ending platform of the module is horizontal $(\theta=0)$, there is an infinite set of possible values for $\left(q_{1}, q_{2}\right)$. In fact, the only condition that leads to $\theta=0$ is $q_{1}=q_{2}$. This case is underlined in red in Figs. 12, 13 and 14.

The second limit case is when the tilt reaches its maximum value $(|\theta|=\pi / 6)$, which happens when $\left|q_{1}-q_{2}\right|=\pi$. In this case, there exists a single solution for $\left(q_{1}, q_{2}\right)$. This case is underlined in magenta in Figs. 12, 13 and 14.

The difference between the aspect areas is that a couple $\left(q_{1}, q_{2}\right)$ in aspect area 1 corresponds to a positive $\theta$ coupled with an arbitrary value of $\phi$. Another couple $\left(q_{1}, q_{2}\right)$ in aspect area 2, bringing the end-effector in the same posture, corresponds to the negative value of $\theta$ and a value of $\phi$ rotated by $\pm \pi$.

Figures 15 and 16 show the same blue path traced in the workspace, a circle with $|\theta|=\pi / 9$. However, the path is traced in aspect area 1 for the first case and in aspect area 2 for the second case. 


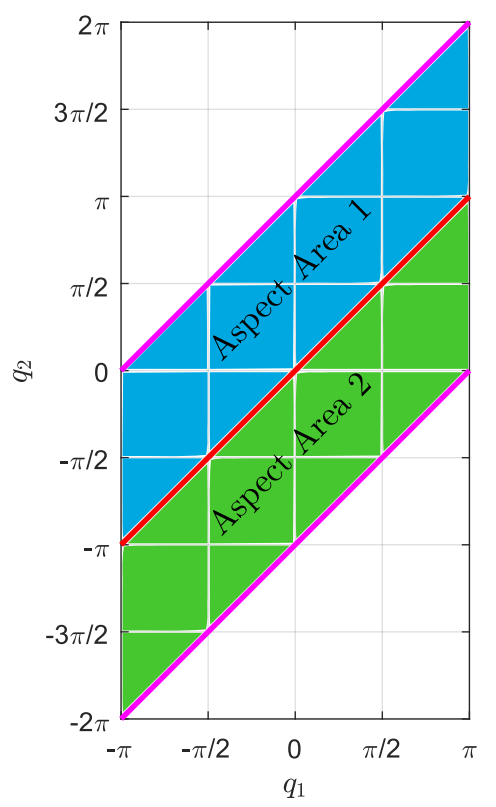

FIGURE 14. ASPECT AREAS OF A MODULE SHOWN IN THE JOINT SPACE
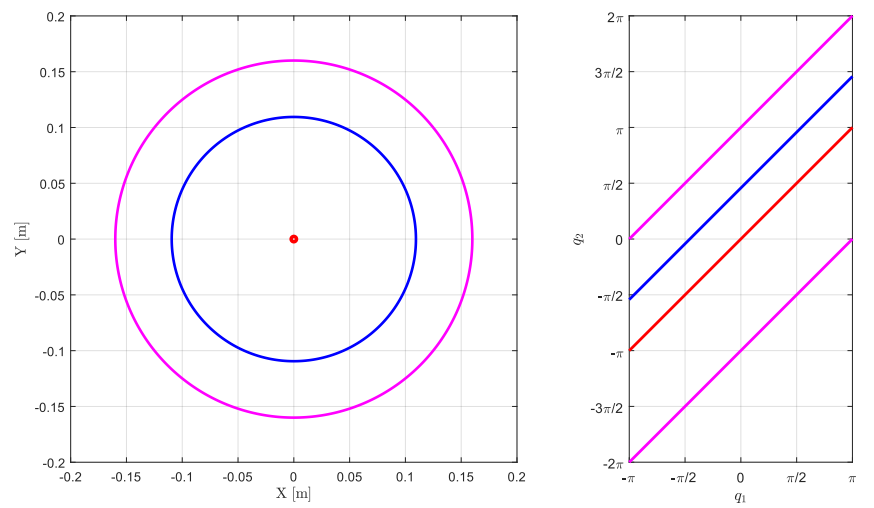

FIGURE 15. THE BLUE PATH IN ASPECT 1, CORRESPONDING TO A TILT OF $20^{\circ}$, IS SHOWN BOTH IN THE WORKSPACE AND IN THE JOINT SPACE

\section{Dexterity}

The condition number $\kappa(\mathbf{J})$ of the kinematic Jacobian matrix of a manipulator and its inverse $k(\mathbf{J})$ are defined in [25] as:

$$
\left\{\begin{array}{l}
\kappa(\mathbf{J})=\left\|\mathbf{J}|||| \mathbf{J}^{-1}\right\| \\
k(\mathbf{J})=1 / \kappa(\mathbf{J})
\end{array}\right.
$$

The index $k$ characterizes the dexterity of the manipulator and is bounded between 0 and 1 . The closer $k$ to 1 , the better the
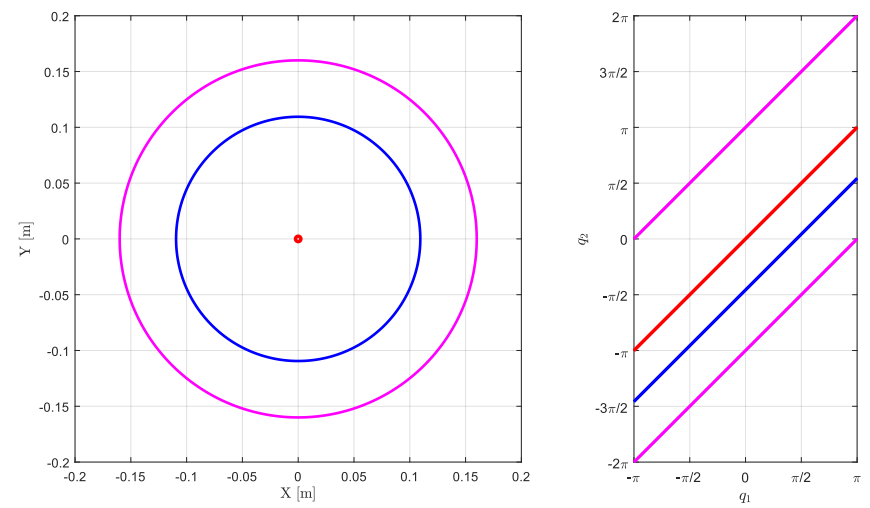

FIGURE 16. THE BLUE PATH IN ASPECT 2, CORRESPONDING TO A TILT OF $20^{\circ}$, IS SHOWN BOTH IN THE WORKSPACE AND IN THE JOINT SPACE

manipulator dexterity. The manipulator reaches an isotropic posture when $k=1$. The closer $k$ to 0 , the worse the manipulator dexterity and the closer it is to a singularity.

In what remains, the kinematic Jacobian matrix $\mathbf{J}_{\omega_{x} \omega_{y}}$, which maps $\left[\dot{q}_{1}, \dot{q}_{2}\right]^{\top}$ to $\left[\omega_{x}, \omega_{y}\right]^{\top}$, is used to test the dexerity of the module accordingly:

$$
\left[\omega_{x} \omega_{y}\right]^{\top}=\mathbf{J}_{\omega_{x} \omega_{y}} \dot{\mathbf{q}}
$$

with

$$
\mathbf{J}_{\omega_{x} \omega_{y}}=\left[\begin{array}{cc}
-\cos \phi \sin \theta & -\sin \phi \\
-\sin \phi \sin \theta & \cos \phi
\end{array}\right] \mathbf{J}_{q_{1} q_{2}}
$$

Figures 17 and 18 show the isocontours of the dexterity index $k$ in the module's workspace and joint space, respectively.

It is apparent that the Nimbl'Bot module has two singularity regions:

$$
\left\{\begin{array}{lll}
\theta=0 & \text { i.e. } & q_{1}=q_{2} \\
|\theta|=\pi / 6 & \text { i.e. } & \left|q_{1}-q_{2}\right|=\pi
\end{array}\right.
$$

On the contrary, the module reaches an isotropic posture when:

$$
\left\{\begin{array}{l}
|\theta|=\arctan \left( \pm \frac{\sqrt{2} \tan \alpha}{2+\tan ^{2} \alpha}\right) \\
\left|q_{1}-q_{2}\right|=\pi / 2
\end{array}\right.
$$




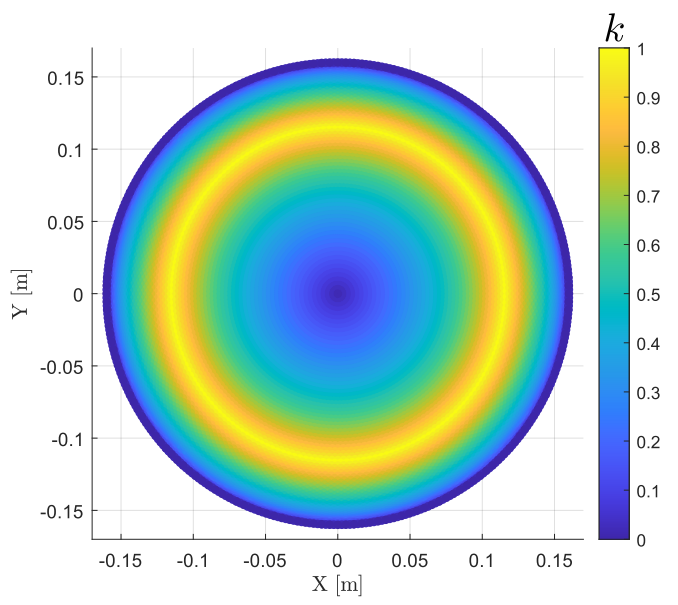

FIGURE 17. DEXTERITY OF THE MODULE SHOWN IN THE CARTESIAN SPACE

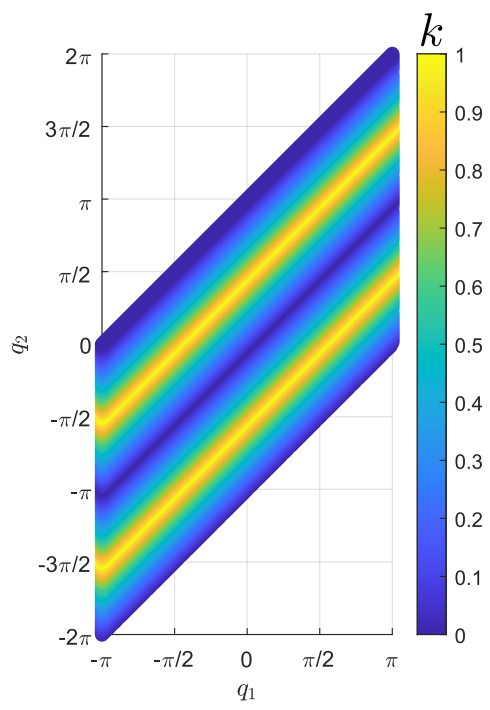

FIGURE 18. DEXTERITY OF THE MODULE SHOWN IN THE JOINT SPACE

\section{WORKSPACE ANALYSIS OF THE NIMBL'BOT ROBOT}

This section introduces a potential design for a machining robot, inspired by a human arm, to show a possible use case for the presented module. This arm has three moving parts, or regions, connected by two links, each part being made of an arrangement of several modules. Each of the three regions in the robot has at least six degrees of freedom, giving more flexibility to the robot. The two links are necessary to increase the manipulator's reachable area of the robot. In this analysis, the length of the two links $l$ is set to 1 meter $(l=1 \mathrm{~m})$.
Figure 19 illustrates the boundaries of the robot workspace. The bold black line represents the workpiece on which the robot has to operate. The complete workspace of the robot is equal to the section shown in Figure 19 rotated about the Z-axis.

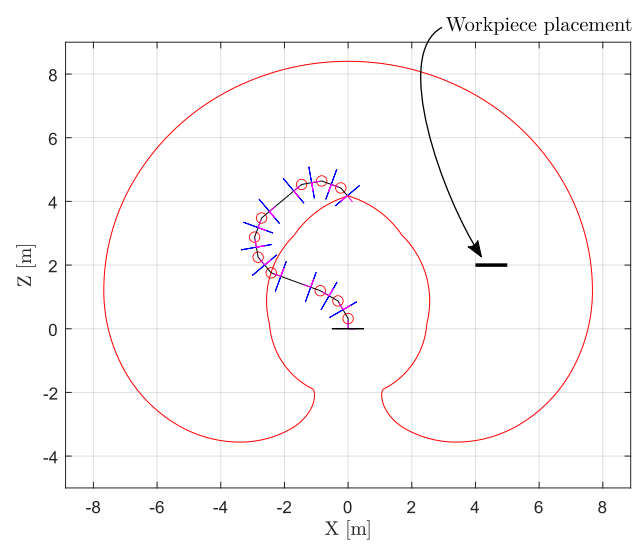

FIGURE 19. BOUNDARY OF THE ROBOT WORKSPACE AND WORKPIECE

\section{CONCLUSIONS AND FUTURE WORK}

This paper described a new two-DoF closed-loop mechanism developed by the company Nimbl'Bot. This module aims to increase the stiffness of serial robots without affecting their workspace and kinematic capabilities. This paper proposed a geometric and kinematic analysis of the novel mechanism. First, the forward and inverse geometric models of the module, the latter amounting to a zero-torsion mechanism, were expressed through the Tilt\&Torsion convention setting the torsion to zero. Then, the kinematic model of the module was derived and its kinematic performance was analyzed based on the dexterity index. Finally, this paper proposed a design of a human-inspired arm actuated by a serial arrangement of ten modules. The workspace of this arm was plotted and evaluated against a typical machining task. The obtained results are promising for the development of an industrial robotic arm actuated by several modules. Future work will address the kinematic control of the robotic arm introduced in the paper to validate experimentally the concept of the actuation module, with a specific focus on machining to show the gain in terms of stiffness and machining accuracy.

\section{ACKNOWLEDGMENT}

This work was supported by the ANRT (Agence Nationale Recherche Technologie) grant CIFRE no 2020/1051 and Nimbl'bot company (https://nimbl-bot.com/) 


\section{REFERENCES}

[1] Cheng, K., 2008. Machining dynamics: fundamentals, applications and practices. Springer Science \& Business Media.

[2] Ji, W., and Wang, L., 2019. "Industrial robotic machining: a review". The International Journal of Advanced manufacturing Technology, 103(1-4), pp. 1239-1255.

[3] Chen, Y., and Dong, F., 2013. "Robot machining: recent development and future research issues". The International Journal of Advanced Manufacturing Technology, 66(9-12), pp. 1489-1497.

[4] Dumas, C., Caro, S., Garnier, S., and Furet, B., 2012. "Workpiece Placement Optimization of Six-Revolute Industrial Serial Robots for Machining Operations". Proceedings of the ASME 2012 11th Biennial Conference on Engineering Systems Design and Analysis, 2, pp. 419-428.

[5] Caro, S., Dumas, C., Garnier, S., and Furet, B., 2013. "Workpiece placement optimization for machining operations with a KUKA KR270-2 robot". In 2013 IEEE International Conference on Robotics and Automation, IEEE, pp. 2921-2926.

[6] Zhang, H., Wang, J., Zhang, G., Gan, Z., Pan, Z., Cui, H., and Zhu, Z., 2005. "Machining with flexible manipulator: toward improving robotic machining performance". Proceedings, 2005 IEEE/ASME International Conference on Advanced Intelligent Mechatronics., pp. 1127-1132.

[7] Takeuchi, Y., Ge, D., and Asakawa, N., 1993. "Automated polishing process with a human-like dexterous robot". In [1993] Proceedings IEEE International Conference on Robotics and Automation, IEEE, pp. 950-956.

[8] Liu, L., Ulrich, B., and Elbestawi, M. A., 1990. "Robotic grinding force regulation: design, implementation and benefits". In Proceedings., IEEE International Conference on Robotics and Automation, IEEE, pp. 258-265.

[9] Pires, J. N., Ramming, J., Rauch, S., and Araújo, R., 2002. "Force/torque sensing applied to industrial robotic deburring". Sensor Review.

[10] Abele, E., Bauer, J., Pischan, M., Stryk, O. v., Friedmann, M., and Hemker, T., 2010. "Prediction of the tool displacement for robot milling applications using coupled models of an industrial robot and removal simulation". In Proc. CIRP 2nd Inter Conf on Process Machine Interactions, Vancouver, Canada.

[11] Schneider, U., Drust, M., Ansaloni, M., Lehmann, C., Pellicciari, M., Leali, F., Gunnink, J. W., and Verl, A., 2016. "Improving robotic machining accuracy through experimental error investigation and modular compensation". The International Journal of Advanced Manufacturing Technology, 85(1-4), pp. 3-15.

[12] Klimchik, A., Pashkevich, A., Wu, Y., Caro, S., and Furet, B., 2012. "Design of calibration experiments for identification of manipulator elastostatic parameters". arXiv preprint
arXiv:1211. 6101.

[13] KUKA Robotics Corporation, 2016. Surface processing with a KR QUANTEC and KUKA.CNC. On kuka.com https://www.kuka.com/en-at/ industries/solutions-database/2016/07/ solution-robotics-sematek. Online; accessed 07 December 2020.

[14] Khalil, W., and Dombre, E., 2004. Modeling, identification and control of robots. Butterworth-Heinemann.

[15] Tanev, T. K., 2000. "Kinematics of a hybrid (parallelserial) robot manipulator". Mechanism and machine theory, 35(9), pp. 1183-1196.

[16] Shammas, E., Wolf, A., Brown, H. B., and Choset, H., 2003. "New joint design for three-dimensional hyper redundant robots". In Proceedings 2003 IEEE/RSJ International Conference on Intelligent Robots and Systems (IROS 2003)(Cat. No. 03CH37453), Vol. 4, IEEE, pp. 3594-3599.

[17] Dufau, L., 2015. Articulated robot arm. https:// uspto.report/patent/app/20180243927. Online; accessed 07 December 2020.

[18] Siciliano, B., 1990. "Kinematic control of redundant robot manipulators: A tutorial". Journal of intelligent and robotic systems, 3(3), pp. 201-212.

[19] Chirikjian, G. S., (1992). "Theory and applications of hyper-redundant robotic manipulators". PhD thesis, California Institute of Technology.

[20] Burdick, J. W., and Chirikjian, G. S., 1991. "Hyperredundant robot mechanisms and their applications". Conference: Intelligent Robots and Systems '91. 'Intelligence for Mechanical Systems.

[21] Carricato, M., 2009. "Decoupled and homokinetic transmission of rotational motion via constant-velocity joints in closed-chain orientational manipulators". Journal of Mechanisms and Robotics, 1(4), p. 041008.

[22] Ginnante, A., 2020. "Geometric and kinematic modeling of Nimbl'Bot robot". Master's thesis, University of Genova Ecole Centrale de Nantes.

[23] Bonev, I., Zlatanov, D., and Gosselin, C., 2002. "Advantages of the modified Euler angles in the design and control of PKMs". 2002 Parallel Kinematic Machines International Conference, pp. 171-188.

[24] Nayak, A., Caro, S., and Wenger, P., 2018. "Comparison of 3 -[pp] s parallel manipulators based on their singularity free orientation workspace, parasitic motions and complexity". Mechanism and Machine Theory, 129, pp. 293-315.

[25] Angeles, J., and López-Cajún, C. S., 1992. "Kinematic isotropy and the conditioning index of serial robotic manipulators". The International Journal of Robotics Research, 11(6), pp. 560-571. 